\title{
Fluorescent ratiometric pH indicator SypHer2: applications in neuroscience and regenerative biology
}

Mikhail E. Matlashov a,b, Yulia A. Bogdanova ${ }^{a, c}$, Galina V. Ermakova ${ }^{a}$, Natalia M. Mishina ${ }^{a, b}$, Yulia G. Ermakovaa, Evgeny S. Nikitin ${ }^{d}$, Pavel M. Balaban ${ }^{d}$, Shigeo Okabe ${ }^{e}$, Sergey Lukyanov ${ }^{\mathrm{a}, \mathrm{b}}$, Grigori Enikolopov ${ }^{\mathrm{f}, \mathrm{g}, \mathrm{h}, \mathrm{l,},}$, Andrey G. Zaraisky ${ }^{\mathrm{a}, \star}$, Vsevolod V. Belousov ${ }^{\mathrm{a}, \mathrm{b},{ }^{*}}$

a Shemyakin-Ovchinnikov Institute of Bioorganic Chemistry, 117997 Moscow, Russia

${ }^{\mathrm{b}}$ Nizhny Novgorod State Medical Academy, 603005 Nizhny Novgorod, Russia

${ }^{c}$ Faculty of Biology, Moscow State University, 119991 Moscow, Russia

d Institute of Higher Nervous Activity and Neurophysiology, 117485, Moscow, Russia

e Department of Cellular Neurobiology, Graduate School of Medicine, University of Tokyo, Tokyo, 113-0033, Japan. ${ }^{\mathrm{f} C o l d ~ S p r i n g ~ H a r b o r ~ L a b o r a t o r y, ~ C o l d ~ S p r i n g ~ H a r b o r, ~ N Y ~ 11724, ~ U S A ~}$

${ }^{9}$ Department of Anesthesiology, Stony Brook School of Medicine, Stony Brook, NY 11794, USA

${ }^{\text {h}}$ Center for Developmental Genetics, Stony Brook University, Stony Brook, NY 11794, USA

'NBIC, Moscow Institute of Physics and Technology, 123182, Moscow, Russia

${ }^{*}$ Corresponding authors at:

1.Shemyakin-Ovchinnikov Institute of Bioorganic Chemistry, 117997 Moscow, Russia

E-mail address: vsevolod.belousov@gmail.com (V.V. Belousov, address regarding SypHer-2 sensor plasmids, synaptic probe targeting and imaging);

2. Cold Spring Harbor Laboratory, Cold Spring Harbor, NY 11724, USA

E-mail address: enik@cshl.edu (G. Enikolopov, address regarding neuronal cultures and imaging);

3. Shemyakin-Ovchinnikov Institute of Bioorganic Chemistry, 117997 Moscow, Russia

E-mail address: azaraisky@yahoo.com (Andrey G. Zaraisky, address regarding Xenopus leavis regeneration experiments). 


\begin{abstract}
Background: SypHer is a genetically encoded fluorescent $\mathrm{pH}$-indicator with a ratiometric readout, suitable for measuring fast intracellular $\mathrm{pH}$ shifts. However, a relatively low brightness of the indicator limits its use.

Methods: Here we designed a new version of $\mathrm{pH}$-sensor - SypHer-2, that has up to three times brighter fluorescence signal in cultured mammalian cells compared to the SypHer.

Results: Using the new indicator we registered activity-associated $\mathrm{pH}$ oscillations in neuronal cell culture. We observed prominent temporal neuronal cytoplasm acidification that occurs in parallel with calcium entry. Furthermore, we monitored $\mathrm{pH}$ in presynaptic and postsynaptic termini by targeting SypHer-2 directly to these compartments and revealed marked differences in $\mathrm{pH}$ dynamics between synaptic boutons and dendritic spines. Finally, we were able to reveal for the first time the intracellular $\mathrm{pH}$ drop which occurs within an extended region of the amputated tail of the Xenopus laevis tadpole before it begins to regenerate.

Conclusions: SypHer2 is suitable for quantitative monitoring of $\mathrm{pH}$ in biological systems of different scales, from small cellular subcompartments to animal tissues in vivo.

General significance: The new $\mathrm{pH}$-sensor will help to investigate $\mathrm{pH}$-dependent processes in both in vitro and in vivo studies.
\end{abstract}




\section{Introduction}

A diversity of synthetic and genetically encoded indicators and active development of fluorescent microscopy in recent years gave powerful tools to image many different parameters in living cells. One of the key parameters in the cells is $\mathrm{pH}$. Not surprisingly, a number of indicators for $\mathrm{pH}$ exist, either synthetic or genetically encoded [1-3]. These $\mathrm{pH}$ probes are of different nature (synthetic [ $\underline{4}, \underline{5}]$ vs. protein $[\underline{6-10}]$ ), color (mostly green $[\underline{4}, \underline{6}, \underline{7}]$ and red $[\underline{9}, \underline{10}]$ ), pKa and readout (ratiometric [ㄴ, $\underline{5}, \underline{7-9}]$ vs. intensiometric $[\underline{6}, \underline{10}]$ ). Of those, genetically encoded sensors provide many benefits, such as a vast range of options for targeting tissues, cells, or organelles [3] and a possibility of expression in transgenic animals [11]. Ratiometric readout offers quantitative measurements devoid of potential artifacts caused by object movement or different expression levels between the parts of the object.

One such pH sensor, SypHer, is a genetically encoded fluorescent ratiometric $\mathrm{pH}$ probe [12]. SypHer is a product of C199S mutation of HyPer, indicator for $\mathrm{H}_{2} \mathrm{O}_{2}$ [13]. The mutation makes HyPer insensitive to hydrogen peroxide while preserving its sensitivity to changes in $\mathrm{pH}$. SypHer has been successfully applied in vitro; however, it's fluorescence is relatively dim, making the use of the indicator somewhat problematic in tissue culture and live animal studies.

Recently an A406V substitution was described for HyPer that significantly increased the dynamic range of the biosensor [14]. For yet unknown reason, the resulting probe, HyPer-2, demonstrates a two to three-fold brighter signal when expressed in mammalian cells (unpublished observation), although purified HyPer and HyPer-2 demonstrate similar brightness [14]. Based on this finding, we decided to improve the reporter features of SypHer using the same mutation. For that, we have introduced C199S substitution into HyPer-2 and tested its performance in various models, ranging from individual synaptic termini in neurons to regenerating Xenopus leavis tadpole fins.

During neuronal activity, large amounts of ions move across the cellular membranes. Within milliseconds intracellular concentrations of $\mathrm{Na}^{+}, \mathrm{K}^{+}, \mathrm{Ca}^{2+}$ and $\mathrm{Cl}^{-}$change significantly [15-17] and maintaining normal homeostasis requires a complex regulation system and high metabolic rate [18]. Not surprisingly, intense neuronal activity causes changes in intracellular $\mathrm{pH}$ in neurons. Numerous studies have shown that prolonged membrane depolarization and calcium entry are accompanied by significant cytoplasm acidification [19-22]. The mechanism of such change in 
cytoplasmic $\mathrm{pH}$ is yet to be determined. One of possible explanations may be related to the activity of the ATP-dependent $\mathrm{Ca}^{2+} / \mathrm{H}^{+}$and $\mathrm{Na}^{+} / \mathrm{H}^{+}$antiport [21]. Another explanation for intracellular acidification would invoke, as the main cause, lactate accumulation resulting from an accelerated glycolysis [20]. In turn, changes in the intracellular $\mathrm{pH}$ affect neuronal activity. For instance, activities of voltage-gated calcium channels, NMDA and GABA receptors, and other key components of the neuronal machinery depend on $\mathrm{pH}$ [23-25]. In addition, neurotransmitter transport is also dependent on the $\mathrm{H}^{+}$gradient across the synaptic vesicular membrane [26]. Finally, in some models of epileptiform activity it was shown that spontaneous seizure-like electric activity duration time may be shortened by intracellular acidification [22, 27]. Therefore, $\mathrm{pH}$ changes may play a significant role as a feedback mechanism for regulating neuronal activity.

Neuronal cells have complex morphological and functional structure and measuring intracellular $\mathrm{pH}$ is not an ordinary task. The use of chemical indicators such as SNARF and BCECF does not allow effective differentiation between, for example, cytoplasm of a dendrite and the content of a dendritic spine, in which critical processes of electric input modulation occur [28]. However, it is possible that $\mathrm{pH}$ changes in fine synaptic structures differ from those in the dendrite given partial isolation of the spines' content from the rest of the cytoplasm.

Changes in $\mathrm{pH}$ and concentration of $\mathrm{H}^{+}$ions may also play an important role in early development and during regeneration. Transport of $\mathrm{H}^{+}$ions across cell membrane attracts special attention because in amphibians it is known to regulate such critical processes as neural induction and tadpole tail regeneration. For example, it has been demonstrated in Xenopus laevis that tail regeneration requires the activity of the V-ATPase $\mathrm{H}^{+}$pump and that enhancing V-ATPase activity induces $\mathrm{H}^{+}$export across cell plasma membranes in regenerating tissues starting $6 \mathrm{~h}$ postamputation [29, 30]. So far, however, $\mathrm{pH}$ dynamics during tail regeneration was studied, to our knowledge, only by indirect approaches, using membrane-voltage-reporter dyes [29-31]. Therefore, detailed data on $\mathrm{pH}$ dynamics during regeneration is lacking. Here, we analyze early $\mathrm{pH}$ dynamics in the Xenopus laevis tails using SypHer-2 $\mathrm{pH}$ sensor and reveal a new phenomenon of a rapid drop of the intracellular $\mathrm{pH}$ occurring within a broad region adjacent to the site of tail amputation. 


\section{Materials and methods}

\subsection{Reagents used}

DMEM, glutamine, HBSS, trypsin-EDTA, penicillin/streptomycin solutions were from PanEko (Russia), heat inactivated FBS, Neurobasal medium, Tyrode solution, B27 supplement, Gluta-Max solution, poly D-lysine, Ca-phosphate transfection kit were from Invitrogen (USA), potassium gluconate, sodium gluconate, BCECF, BAPTA-AM, bicuculline, nigericin, monensin, tetrodotoxin, bafilomycin were from Sigma, X-tremeGene 9 was from Roche (France). Glass bottom dishes were from Mattek. NIH/3T3 cells were from ATCC, HeLa Kyoto cells were provided by EMBL. R-GECO encoding vector was from Addgene (https://www.addgene.org/32465/), pHyPer-N and pHyPer-C vectors was from Evrogen. pCS2 vector (Xenbase 1221270) was used for RNA preparation.

\subsection{Constructs preparation}

To introduce a C199S mutation into HyPer-2, we amplified two fragments using two pairs of primers: $\quad 5$ '-tccgctagcgccaccatggagatggcaagccag-3', 5'-atcgcgcaaagagtgaccatct-3', and 5'agatggtcactcttgcgcgat-3', 5'-atcaagcttttaaaccgcctgtt-3', and pHyPer-2-C plasmid as a template. This was followed by another round of PCR, using the PCR fragments obtained and $5^{\prime}$ tccgctagcgccaccatggagatggcaagccag-3' and 5'-atcaagcttttaaaccgcctgtt-3' primers to produce the complete sensor construct. The final sensor-carrying PCR fragment was cloned into the pHyPer-C vector using Nhel and Hindlll restriction sites, forming pSypHer-2-C vector.

For SypHer-2-Homer1 construct preparation we used 5'- ttcgaattcatgggggaacaacctatc-3' and 5'- ggtggatccttagctgcattctagtagc-3' primers, with Homer1 containing vector pGFP-PSD-Zip45 [32] as a template, and cloned the resulting fragment into the pSypHer-2-C vector using EcoRI and BamHI restriction enzymes.

For Synaptophysin-SypHer-2 construct preparation we first subcloned SypHer-2 to pHyPer-N vector using 5'-tccggacttaccggtgccaccatggagatggcaagccag-3' and 5'-atcgcggccgctttaaaccgcctgtt-3' primers and BamHI/Notl restriction enzymes. To obtain a Synaptophysin fusion construct, we amplified the fragment of Synaptophysin using 5'-tccgctagcatggacgtggtgaatcag-3' and 5'gagctcgagcatctgattggagaagg-3' primers and pSynaptophysin-GFP [푸 vector as a PCR template, and cloned the fragment into the pSypHer-2-N vector using Nhel and Xhol restriction enzymes. The resulting structure of each construct was confirmed by sequencing. 
To generate pCS2-SypHer-2 vector we amplified SypHer-2 fragment using 5'-ccgggatcc gccaccatggagatggcaagccag-3' and 5'-ggatctagattaaaccgcctgtttaaaac-3' pair of primers and subcloned it into pCS2 vector using BamHI and Xbal restriction enzymes.

\subsection{Cell cultures and transfection}

HeLa-Kyoto and $\mathrm{NIH} / 3 \mathrm{~T} 3$ cells were cultured in DMEM supplemented with $10 \%$ FBS at $37^{\circ} \mathrm{C}$ in a $5 \% \mathrm{CO}_{2}$ incubator. Cells were split every second day and seeded on $35 \mathrm{~mm}$ glass bottom dishes. Twenty-four hours later cells were transfected with a mixture of $1 \mu \mathrm{g}$ vector DNA and $3 \mu \mathrm{I}$ XtremeGene 9 transfection reagent per dish according to the manufacturer's recommendations.

Mixed mouse primary embryonic hippocampal neuronal cell culture was prepared as indicated in [34], with the protocol for animal handling approved by the ethical committee of the Institute of Bioorganic Chemistry RAS. Pregnant C57BI/6 mice were used at E17 and embryos were anesthetized in ice cold HBSS with low $\mathrm{Mg}^{2+}$ and $\mathrm{Ca}^{2+}$, brains were extracted, hemispheres separated from meninges and hippocampi collected in ice cold HBSS. The hippocampi were incubated in $0.05 \%$ trypsin/EDTA solution for 15 minutes at $37^{\circ} \mathrm{C}$, washed three times in warm DMEM supplemented with 10\% FBS and $500 \mathrm{mM}$ glutamine (plating medium), and carefully triturated with a $1 \mathrm{ml}$ tip for 10 times. Cells were seeded on poly-D-lysine covered $35 \mathrm{mM}$ glass bottom dishes, at $15^{*} 10^{4}$ cells in $100 \mu$ of plating medium. 1-2 hours after plating, medium was changed for $2 \mathrm{ml}$ Neurobasal medium supplemented with B27, 10\% heat inactivated FBS and 2 $\mathrm{mM}$ GlutaMax. Once in three days $1 / 3$ of medium was exchanged to a fresh Neurobasal medium. On the 5th day in vitro, cells were transfected with vector DNA using a Ca-Phosphate transfection kit. One hour before transfection, growth medium was removed to a $50 \mathrm{ml}$ tube (conditioned medium) and $1.5 \mathrm{ml}$ DMEM was added to the dishes. For each dish, $100 \mu \mathrm{l}$ of DNA-CaCl 2 mixture (total $4 \mu \mathrm{g}$ DNA) was added dropwise to $100 \mu \mathrm{l}$ of $2 x$ HBSS while vortexing at low speed. After 25 min incubation, $200 \mu$ of the solution was added to plates which were then incubated for $15 \mathrm{~min}$ at $5 \% \mathrm{CO}_{2}$ incubator. After transfection, neurons were washed 2 times with DMEM, then filled with the conditioned Neurobasal medium.

\subsection{Cell culture imaging and analysis}

HeLa Kyoto and NIH/3T3 cells were placed into an environmental chamber in HBSS solution at $37^{\circ} \mathrm{C}$ and imaged using a Leica CTR6500 wide-field microscope equipped with a 20x air objective and HCX PL APO Ibd.BL 63X 1.4NA oil objective, TX2 filter cube and CFP/YFP FRET filter cube (dichroic 440/520) coupled to CFP/YFP FRET external filter wheel set. Neuronal cells were 
imaged 14-20 days after plating in a Tyrode solution, supplemented with $20 \mathrm{mM}$ D-Glucose and 20 mM Hepes pH 7.35 with 3 sec time laps interval. SypHer fluorescence was sequentially excited at 427 and $504 \mathrm{~nm}$ using 427/10 and 504/12 band-pass excitation filters; emission of the indicator was collected using a 525/50 band-pass emission filter. R-GECO fluorescence was excited via 561/10 band-pass filter and 610/50 filter was used for emission.

SypHer-2-Homer- and Synaptophysin-SypHer-2-expressing neurons were imaged with Leica SP2 confocal microscope in a line scan mode at 500 lines per second with exposure time 2 ms per line of 128 pixels.

Intracellular $\mathrm{pH}$ calibration was performed as described in [12]. High $\mathrm{K}^{+}$calibration buffer contained $5 \mu \mathrm{M}$ nigericin, $5 \mu \mathrm{M}$ monensin, $130 \mathrm{mM}$ potassium gluconate, $20 \mathrm{mM}$ sodium gluconate, $0.5 \mathrm{mM} \mathrm{MgCl}_{2}, 0.2 \mathrm{mM}$ EGTA, and $30 \mathrm{mM}$ Tris (pH 8.0-9.0), HEPES (pH 6.9 - 7.9), or MES (pH 6.0 - 6.8). For each $\mathrm{pH}$ point, adjacent cells were washed and incubated for 3-4 minutes in a corresponding calibration buffer at $37^{\circ} \mathrm{C}$.

Time series were analyzed using ImageJ software (NIH). For pH dynamics calculation, stacks corresponding to $427 \mathrm{~nm}$ and $504 \mathrm{~nm}$ excitation bands of SypHer were converted to 32 bit after background subtraction. A 427-nm stack was thresholded to remove pixel values from the background. A 500-nm stack was divided by the corresponding 427-nm stack frame by frame. Time course of SypHer fluorescence was calculated for regions of interest (ROI) inside the imaged cell. All graphs were made in OriginPro 8 (OriginLab); calibration curves were obtained by sigmoidal fitting. Line scan data for synaptic constructs was processed with a fast Fourier transform filter.

\subsection{Embryo manipulation}

Synthetic mRNA encoding SypHer was generated with an SP6 Message Machine Kit (Ambion) from pCS2-SypHer-2 plasmid linearized with Notl. To obtain tadpoles containing SypHer in tail cells, we injected embryos at 2-4 cell stages as described in ref. [35] with SypHer synthetic mRNA (100 pg/blastomere) and grew them to the tadpole stage. Upon tadpoles reaching desired stages, they were inspected using fluorescent stereomicroscope M205 (Leica), and those demonstrating a more uniform distribution of SypHer signal in their tails were selected for further monitoring of the SypHer ratio.

\subsection{Monitoring of $\mathrm{pH}$ dynamics in tadpoles by SypHer ratio measurement}


To determine the SypHer ratio, a succession of paired photographs of tail tips was obtained during $1 \mathrm{~h}$ after amputation using Violet and EGFP3 filter sets, with $30 \mathrm{sec}$ time-lapse interval between each pair of photos. Then, mean SypHer ratio in a 30-microns width frame, which was stepwise shifted for the distance of 30 microns at each step in the distal to proximal direction from the amputation edge, was calculated for each time-lapse point. Together, this produced a set of specific curves describing SypHer ratio dynamics at each of the analyzed ten positions along the distal to proximal axis. Finally, the obtained SypHer ratio curves were transformed into $\mathrm{pH}$ curves by using a calibration plot that relates specific values of SypHer ratio to corresponding values of $\mathrm{pH}$. This calibration plot was generated beforehand by measuring the SypHer ratio directly in tails of stage 42 tadpoles permeabilized by ionophores nigericin and monensin at known $\mathrm{pH}$ values as indicated above (see Cell culture imaging and analysis).

\section{Results}

\subsection{Investigating SypHer-2 $\mathrm{pH}$ sensitivity and brightness in cell cultures}

Brightness is a crucial characteristic of fluorescent proteins used for live cell imaging because higher brightness allows lowering the excitation light intensity and exposure time, therefore minimizing phototoxic effects. We have noticed that despite similar brightness of purified HyPer [13] and HyPer-2 [14] $\mathrm{H}_{2} \mathrm{O}_{2}$ sensors, HyPer-2 is visibly brighter than HyPer when expressed in mammalian cell cultures. HyPer-2 differs from HyPer by a single amino acid (A406V) residing in the dimerization interface of the OxyR part of the sensor [14]. We therefore hypothesized that the same mutation could improve in situ brightness of the SypHer (HyPer-C199S) pH indicator. To obtain the SypHer-2 probe, we introduced a C199S substitution into HyPer-2. This mutation makes the probe insensitive to $\mathrm{H}_{2} \mathrm{O}_{2}$, but does not affect $\mathrm{pH}$-sensitivity of the chromophore [13]. To determine $\mathrm{pH}$-sensitivity of the indicator in cultured cells we measured its F500/F420 ratio in HeLa Kyoto cells at pH $6.0-9.0$ in a buffer set containing ionophores nigericin and monensin (Fig 1. A,B). pH sensitivities of SypHer and SypHer-2 appeared to be identical (Fig. 1B). The sensor's F500/F420 ratio increases more than 10 fold upon $\mathrm{pH}$ change from 6.0 to 9.0 and has a linear slope at the $\mathrm{pH}$ range $7.5-8.5$ with the pKa 8.1, which is similar to SypHer and different HyPer versions [12-14, 36].

To determine the effect of $\mathrm{A} 406 \mathrm{~V}$ substitution on the brightness of the indicator in mammalian cells, we transfected two different cell lines, HeLa-Kyoto and $\mathrm{NIH} / 3 \mathrm{~T} 3$, with an equal amount of $\mathrm{C} 1$ vector encoding either SypHer or SypHer-2 under the same CMV promoter. The fluorescence intensity of SypHer-2 in the resting cells $24 \mathrm{~h}$ post-transfection appeared to be about two-fold 
higher than that of SypHer in HeLa-Kyoto cells and up to three-fold higher in NIH/3T3 fibroblasts (Fig. 1C, D). Given that insufficient brightness is a serious disadvantage for most of the cpYFPbased indicators, we considered the two-to-three fold increase in the fluorescence intensity of SypHer-2 as a significant advance.

The improved brightness of SypHer-2 compared to SypHer could be due to a number of possible reasons: higher protein synthesis rate, faster chromophore maturation, lower protein degradation rate or higher physical brightness (product of quantum yield and extinction). In order to discriminate between these possibilities we performed time lapse imaging of cells transfected with either SypHer or SypHer-2, treated or non-treated with protein synthesis inhibitor cycloheximide. Either higher protein synthesis rate or faster chromophore maturation would result in faster dynamics of fluorescence increase in cycloheximide-free cells. Lower protein degradation rate would result in slower decrease in fluorescence upon cycloheximide treatment. At the beginning of the time lapse series SypHer-2 expressing cells were two-fold brighter compared to SypHer cells (data not shown). Fluorescence of both probes gradually increased up to two-fold within the next 18 hours with no difference between the sensors as shown on the normalized graph (Fig 1. E). Upon cycloheximide treatment both probes demonstrated equally perfect stability with almost no change in fluorescence level over 18 hours of the experiment. Therefore improved brightness of SypHer-2 is not a result of altered synthesis, maturation or degradation. Most plausible possibility is the higher physical brightness of SypHer-2 in mammalian cells. Unfortunately, this parameter cannot be assessed in situ and the reason for the improved brightness remains yet unidentified.

\subsection{Registration of the spontaneous $\mathrm{pH}$-oscillations in a primary neuronal culture}

$\mathrm{pH}$ values in neurons can vary in an activity-dependent manner [37-40]. However, in most of the neuronal models analyzed for changes in $\mathrm{pH}$, neurons were subjected to strong stimuli, such as depolarization elicited by an electrode on a minute time scale or by externally added NMDA, $\mathrm{K}^{+}$ ions, or other stimulants [21, 39]. Little data exists on whether $\mathrm{pH}$ fluctuates in more physiological settings, where depolarization events are brief [41].

We therefore applied SypHer-2 for the examination of $\mathrm{pH}$ changes during spontaneous neuronal activity, induced by GABA-receptor blockade. We co-transfected cultured primary mouse hippocampal neurons with SypHer-2 to measure the intracellular $\mathrm{pH}$ and a genetically-encoded calcium indicator R-GECO [느] to monitor intracellular $\mathrm{Ca}^{2+}$ levels (Fig.2). 2-3 weeks after isolation cultured neurons formed a network demonstrating spontaneous neuronal activity which was further facilitated by $50 \mu \mathrm{M}$ bicuculline, an inhibitor of neuronal GABA receptors. For quantitative $\mathrm{pH}$ 
evaluation, after each experiment SypHer-2 signal in each cell was calibrated using reference buffer solutions with $\mathrm{pH}$ 7.1-7.5 (Fig. 2D).

Using SypHer-2 we were able to register the spontaneous $\mathrm{pH}$ oscillations in neuronal culture (Fig. 2). Basal pH value in neuronal cytoplasm was 7.4 - 7.5 which corresponds well to the previously reported values in rat hippocampal neurons [22] but is significantly higher than was later reported by other authors $(\mathrm{pH}$ 6.8) [40]. Simultaneous registration of SypHer and R-GECO signals showed that each transient acidification event $(0.05-0.1 \mathrm{pH}$ units) occurred simultaneously with calcium entry and was followed by return of both $\mathrm{Ca}^{2+}$ and $\mathrm{pH}$ values to the initial levels (Fig. $2 A, B, C)$. Both calcium entry and the $\mathrm{pH}$ changes were completely abolished by $1 \mathrm{mM}$ BAPTA-AM or $100 \mathrm{nM}$ TTX (Fig. 2B, C) confirming that the both processes depend on neuronal activity. However, because depletion of intracellular calcium by BAPTA-AM probably disrupts spontaneous electrical activity, it is not clear whether calcium entry is directly involved in cytoplasm acidification. To verify that SypHer-2 signal changes indeed correspond to the $\mathrm{pH}$ oscillations, we incubated RGECO-transfected neurons with $10 \mu \mathrm{M}$ of a pH-sensitive synthetic dye BCECF-AM (Fig. 2E). BCECF fluorescence excited by $504 \mathrm{~nm}$ light demonstrated similar $\mathrm{pH}$ profiles confirming $\mathrm{pH}$-driven nature of the SypHer-2 ratio changes. Therefore, SypHer-2 is suitable for dynamic $\mathrm{pH}$ monitoring in neurons.

\subsection{Registration of the $\mathrm{pH}$ in pre- and postsynaptic termini}

Synapses are functional units of neuronal activity. Being spatially distant from the cell body, pre- and postsynaptic termini host strictly localized signaling and metabolic processes [43]. In fact, ionic homeostasis, including proton concentrations as well as many other activities might be separately regulated in synaptic boutons vs. dendritic spines vs. cell body. Therefore it would be of great importance to be able to monitor $\mathrm{pH}$ at the level of individual synapses. This task is theoretically achievable using ultrafast imaging of a very bright dye, when the acquisition speed is high enough to capture an image at a time scale comparable with the rate of protons and the probe diffusion to and from a synaptic terminal. However, achieving clear synapse vs. process signal separation is a highly challenging task, especially during live cell imaging where spatial resolution is usually limited to avoid bleaching, reduce phototoxicity and achieve reasonable frame rate. Alternative approach would be to target the probe to pre- or postsynaptic termini. In this case signal would be initially restricted exclusively, or to large extent, to synapses. This task can be accomplished by fusing genetically encoded sensors with a protein that specifically localizes to the compartment of interest. We took advantage of the protein nature of SypHer-2 and fused it to proteins localized to post- or presynaptic termini. To target SypHer-2 to the dendritic spines, we 
fused it to postsynaptic protein Homer1, as GFP-Homer1 fusion has been used previously to visualize postsynaptic structures $[\underline{32}, \underline{44}]$. Substituting GFP for SypHer-2 allowed us to target the recombinant $\mathrm{pH}$ probe to the postsynaptic density. Expression of SypHer-2-Homer1 was visible after 10 days in culture as small dots covering neuronal body and dendrites (Fig. 3A and Fig. S1, Supplementary material), similar to the GFP-Homer1 pattern [32]. Note that punctate distribution of SypHer2-Homer1 was not detected at early stages (days 3-9) in culture. This is consistent with the fact that synapses become mature at late stages of culturing, when maturing neurons form a network. Axons did not contain SypHer-2-Homer1 protein. Overall, the pattern of SypHer-2-Homer1 distribution was undistinguishable from that of GFP-Homer1 (Fig. S2, Supplementary material, compare to Fig. 3A upper row). To visualize presynaptic $\mathrm{pH}$ changes, we targeted SypHer-2 to synaptic boutons by fusing it to Synaptophysin (Sph) via a 22 amino acid-long flexible polypeptide linker in order to anchor it on the cytoplasmic side of synaptic vesicles. Similar to SypHer-2Homer1, Synaptophysin-SypHer-2 fusion was only visible after 10 days in culture (Fig. 3C and Fig. S1, Supplementary material). The spatial distribution of the probe was very similar to the previously demonstrated pattern of Sph distribution in neurons [은, $\underline{40}$. Namely, fluorescent puncta were only visible in axons, but not in the dendrites and cell bodies indicating proper localization (Fig. 3A). Note that in our experiments we did not perform co-localization analysis of targeted SypHer with pre- and postsynaptic markers. However, predictive expression patterns together with literature data allow us to state with a high level of confidence that the sensors localize correctly.

In both synaptic compartments we observed activity-associated pH oscillations (Fig. 3B, D). However, we observed marked difference in the shape of the $\mathrm{pH}$ response between the two compartments. Whereas the amplitude of presynaptic $\mathrm{pH}$ oscillations was similar to that in the cell body (up to $0.1 \mathrm{pH}$ units), $\mathrm{pH}$ shifts in the dendritic spines of neurons from the same preparation were larger (up to $0.2 \mathrm{pH}$ units). The pre- and postsynaptic termini also differed significantly in the shape of the transition from acidification to compensating alkalinization. In presynaptic termini, acidification-to-alkalinization transition was gradual, whereas in the dendritic spines it was sharp and was followed by a very rapid realkalinization phase.

The higher amplitude of $\mathrm{pH}$ oscillations in the dendritic spines compared to cell bodies and synaptic boutons could stem from higher $\mathrm{Ca}^{2+}$ load of this compartment during neuronal activity. We co-transfected the neurons with R-GECO Ca ${ }^{2+}$ sensor and either SypHer-2-Homer1 or non-targeted SypHer-2. As R-GECO fluorescence was too low to detect $\mathrm{Ca}^{2+}$ changes in the axons, we compared $\mathrm{Ca}^{2+}$ and $\mathrm{pH}$ dynamics in the dendritic spines with those in the cell bodies, where the amplitude of $\mathrm{pH}$ changes was similar to the synaptic boutons. Indeed, the amplitude of R-GECO signal changes in the dendritic spines was much higher compared to the cell bodies (Fig. S3, 
Supplementary material). Inhibition of $\mathrm{Ca}^{2+}$ ATPases responsible for $\mathrm{Ca}^{2+}$ efflux from the cytoplasm by $500 \mu \mathrm{M} \mathrm{La}{ }^{3+}$ (plasma membrane ATPases) or $10 \mu \mathrm{M}$ thapsigargin (endoplasmic reticulum ATPase SERCA) did not lead to changes in $\mathrm{pH}$ dynamics (Fig. S4, Supplementary material). Therefore $\mathrm{Ca}^{2+} / \mathrm{H}^{+}$exchange is not a cause of cytoplasmic acidification upon $\mathrm{Ca}^{2+}$ increase in our model.

Would $\mathrm{pH}$ and $\mathrm{Ca}^{2+}$ responses differ in spontaneous neuronal activity vs. sustained stimulation model? In order to induce sustained neuronal depolarization we added $30 \mathrm{mM} \mathrm{KCl}$ to the media. $\mathrm{Ca}^{2+}$ response was transient and similar in the amplitude and duration to that in spontaneous activity model (Fig. S5, Supplementary material). However, the $\mathrm{pH}$ response differed: $\mathrm{KCl}$-induced transient alkalinization phase followed by $\mathrm{Ca}^{2+}$ entry and deep ( 0.4 units) and sustained acidification of the spines. Therefore temporal vs. sustained neuron stimulation is reflected in temporal vs. sustained acidification of the cytoplasm.

\subsection{Early $\mathrm{pH}$ changes in Xenopus laevis regeneration}

By using SypHer-2, we were able to show pH dynamics in cultured cells at the level of subcellular structures, such as synapses. Next, it was important to determine whether ratiometric readout of SypHer-2 would be suitable for monitoring $\mathrm{pH}$ and producing quantitative information on the $\mathrm{pH}$ changes in multicellular systems at the tissue scale.. To examine SypHer-2 performance in the whole-organism imaging we turned to early stages of vertebrate regeneration, namely tail regeneration in Xenopus laevis tadpoles. $\mathrm{pH}$ plays a vital role in the regeneration process. It was shown that the activity of the V-ATPase $\mathrm{H}^{+}$pump is required for tail regeneration and that this activity induces $\mathrm{H}^{+}$pumping through cell plasma membranes in regenerating tissues at least by $6 \mathrm{~h}$ after tail amputation [29, 31]. However, what happens with $\mathrm{pH}$ at the early stages of Xenopus tadpole tail regeneration, e.g. within first minutes after amputation, remains unknown.

To determine $\mathrm{pH}$ changes at the early stages of tail regeneration, we injected Xenopus embryos with SypHer-2 mRNA at the 2-4 cells stage. Then, tadpoles of desired age, which demonstrated detectable and uniform fluorescence of SypHer-2 throughout tail tissues, were selected, their tails amputated and SypHer-2 signal was recorded at ten sequential positions distally from the amputation site (see Materials and Methods). We found a rapid drop of $\mathrm{pH}$ after amputation at each of the ten positions where $\mathrm{pH}$ were monitored, i.e., within first 300 microns from the tail amputation edge (Fig. 4A). Interestingly, the minimal $\mathrm{pH}$ value at each of these ten levels was reached very rapidly, within 3-5 min after amputation. The magnitude of the $\mathrm{pH}$ drop in the tissue decreased with distance from the edge of amputation. The value of $\mathrm{pH}$ decrease reached 0.5 unit on average at the starting point ( $15 \mu \mathrm{m}$ from the edge; left pair of bars on Fig. 4B) and declined 
to zero by the distance of $1000 \mu \mathrm{m}$ from the edge (not shown). Notably, we observed no differences in $\mathrm{pH}$ dynamics between the embryos containing different amount of SypHer mRNA. This demonstrates the advantage of the ratiometric readout and validates SypHer-2 for $\mathrm{pH}$ measurements at the tissue level.

What are the mechanisms that might be responsible for the descending and ascending branches of the $\mathrm{pH}$ curves we obtained? V-ATPase was reported to be one of the pumps that generate the $\mathrm{H}^{+}$flux at later stages of regeneration. We sought to determine whether the same pump could be responsible for the acidification-to-alkalinization switch and $\mathrm{pH}$ increase reported by SypHer-2 during the earliest phase of regeneration.. Indeed, addition of bafilomycin, a specific inhibitor of V-ATPase shortly before tail amputation did not affect rapid acidification, but prevented the subsequent alkalinization phase (Fig. 4C).

Next, we investigated whether the observed drop of the intracellular $\mathrm{pH}$ after amputation might result from $\mathrm{H}^{+}$influx from the intercellular fluid to the cytoplasm. One prediction from this model is that an increase in $\mathrm{pH}$ in the intercellular fluid should result in a decrease of the magnitude of the $\mathrm{pH}$ drop after amputation. Indeed, we observed this effect in tadpoles that were preincubated in the solution with $\mathrm{pH} 8.2$ for $2 \mathrm{~h}$ before amputation. The $\mathrm{pH}$ drop in these tails was up to two-fold less compared to untreated control (Fig. 4B, D). Notably, tadpoles exposed to alkaline pH demonstrated reduced capacity for tail regeneration (compare image of a normally regenerating tail on Fig. 4A with the corresponding image on Fig. 4D).

Xenopus tadpoles lose the capacity to regenerate their amputated tails between stages 45 and 48 , in the so called refractory period [45]. Given this fact, along with our data showing the effect of $\mathrm{pH}$ on tail regeneration, we sought to monitor the $\mathrm{pH}$ dynamics in tails amputated during the refractory period. In particular, we were interested to know whether the initial $\mathrm{pH}$ drop will be less pronounced during this period. Indeed, we found that the magnitude of the $\mathrm{pH}$ drop was less in tails amputated at stage 46 than at stage 42 (Fig. 4B, E). The difference was similar to that caused by alkaline $\mathrm{pH}$ during "regenerating" stage 42 (Fig. 4D). The only difference was in the initial $\mathrm{pH}$ value before the amputation, which was approximately $0.1 \mathrm{pH}$ unit higher in stage 42 tails.

\section{Discussion}

Here we describe SypHer-2, a genetically encoded ratiometric $\mathrm{pH}$ indicator of improved brightness. SypHer-2 shows 2-3 times higher fluorescence in mammalian cells than the previous version, SypHer. SypHer-2 has a ratiometric readout that makes measurements independent of the indicator concentration and cytoplasm volume change, which is particularly useful for long time 
measurements. The indicator works in a broad $\mathrm{pH}$ range with the pKa value of 8.1 , which is slightly more alkaline compared to cytoplasmic $\mathrm{pH}$. The ratiometric signal allows accurate measurement of $\mathrm{pH}$ in the 7.0 - 7.4 range, where SypHer-2 ratio changes approximately two-fold.

To generate SypHer-2, we introduced an $\mathrm{A} 406 \mathrm{~V}$ point mutation previously used to improve the dynamic range of HyPer, an $\mathrm{H}_{2} \mathrm{O}_{2}$ sensor from which SypHer is derived [12]. We noticed higher relative brightness of HyPer-2 compared to HyPer [13] and HyPer-3 [36] in the mammalian cell culture. All three probes, HyPer, HyPer-2 and HyPer-3 demonstrate similar brightness when isolated from E.coli, and we do not know the reason for the superior brightness of HyPer-2. Indeed, the A406V mutation which converts HyPer into HyPer-2, appears to be beneficial for SypHer as well, resulting in the SypHer-2 probe. Our attempt to determine the nature of improved brightness of SypHer-2 by analyzing its synthesis and degradation dynamics did not reveal any detectable differences compared to SypHer. We assume that some unidentified parameters in eukaryotic cells determine higher physical brightness of SypHer-2. Notably, both SypHer and SypHer-2 demonstrate superior protein stability in the mammalian cells. Upon inhibition of protein synthesis the cells expressing both probes demonstrate stable level of fluorescence signal at least for the next $16 \mathrm{~h}$.

A number of genetically encoded ratiometric $\mathrm{pH}$ indicators consisting of a single polypeptide are currently available, differing in properties such as excitation and emission wavelength maxima, pKa value, etc. [3]. One such indicator is pHluorin-2 [46]. Its signal is the ratio between the sensor's fluorescence intensity at $508 \mathrm{~nm}$ when excited at $395 \mathrm{~nm}$ or at $475 \mathrm{~nm}$. Another ratiometric $\mathrm{pH}$ indicator previously developed is $E^{2}$ GFP [8]. Its signal is the ratio between the fluorescence intensity at $532 \mathrm{~nm}$ when excited at 488 or $458 \mathrm{~nm}$. Although the pKa values of $E^{2}$ GFP and pHluorine-2 are close to 6.9 versus 8.1 for SypHer-2, within $\mathrm{pH}$ range 7.0 to 7.4 , the $\mathrm{E}^{2}$ GFP ratio changes about 1.5-fold and pHluorin ratio changes even less [47], compared to the 2.0-fold change for SypHer-2. On the other hand, both pHluorin-2 and $E^{2}$ GFP have bright fluorescence comparable to EGFP. E ${ }^{2}$ GFP is reported to have a higher quantum yield (about 0.5) compared for SypHer-2 (about 0.1 for HyPer-derived proteins [36]). However, it should be noted that SypHer-2 demonstrates increased brightness in cell culture, but not in cell lysates, therefore, a true comparison should consider brightness in cells. Thus, while at pH 7.0 - 7.4 E²GFP and pHluorin-2 have somewhat better parameters than SyhPer-2 if brightness of indicator is crucial, SypHer-2 possesses a better dynamic range and has a significant advantage at more alkaline $\mathrm{pH}$ values.

$\mathrm{pH}$ is a fundamental parameter of cellular homeostasis. It would not be overstating to say that all processes in cells are $\mathrm{pH}$-dependent. However, for some systems, like neurons, studies of $\mathrm{pH}$ 
are limited. Historically, neurons are viewed in the context of their electrical activity. Therefore, most studies of neurons have been performed using either electrophysiological methods or optical imaging of the most relevant parameters of excitable cells: plasma membrane potential and $\mathrm{Ca}^{2+}$ influx. In classical electrophysiological approaches to single cell analysis, such as whole cell patch clamp, the cytoplasm is replaced with buffered saline from the pipette [48]. Therefore $\mathrm{pH}$ gets clamped and that, in our view, can distort the true picture of neuronal physiology since we show that the activity of neurons is accompanied by $\mathrm{pH}$ changes. Regarding optical imaging of neurons, lack of substantial amount of data on various parameters apart from voltage and $\mathrm{Ca}^{2+}$ also supports a general view of the neuron as an electrical cable, ignoring a host of cellular features. We therefore used our new probe for $\mathrm{pH}$ monitoring in neurons. For imaging we have chosen a model of spontaneous neuronal activity in mature dissociated hippocampal neuronal cultures [49]. When maintained in culture for two weeks and longer, neurons on culture dish form a network which demonstrates periodic firing. Action potential waves spread through the entire network with a frequency of several events per minute. The firing can be facilitated by inhibiting inhibitory neurons in the network. We considered this model of spontaneous firing as the most relevant because it does not involve artificially strong stimulation of the neurons using, for example, trains of electrical stimulations with durations of seconds [19].

$\mathrm{pH}$ changes induced by spontaneous activity in hippocampal slice cultures have previously been described using other genetically encoded indicators: deGFP4, E²GFP and Cl-sensor [22]. In this study two models of epileptiform activity were used: the low $\mathrm{Mg}^{2+}$ and the low $\mathrm{Cl}^{-}$models. All indicators tested were ratiometric and were able to monitor $\mathrm{pH}$-dynamics quantitatively, registering 0.1-0.3 pH units changes during seizure-like activity; however, it was noted that deGFP4 indicator suffered from relatively low signal to noise ratio, and the $\mathrm{Cl}$-sensor was naturally also sensitive to $\mathrm{Cl}^{-}$-transients. Interestingly, the $\mathrm{pH}$ dynamics differed in temporal properties between the models, with neurons in low $\mathrm{Cl}^{-}$medium displaying faster changes in intracellular $\mathrm{pH}$. However, the authors didn't apply the indicators for measuring $\mathrm{pH}$-dynamics in synaptic compartments.

In our experiments we used GABA receptor blockade model of epileptiform activity, that does not require any changes in the medium ion composition, which might affect subtle cellular reactions. Using SypHer-2 we were able to register electrical activity-induced $\mathrm{pH}$ oscillations. Simultaneous monitoring of $\mathrm{Ca}^{2+}$ in the cytoplasm revealed that the drop in $\mathrm{pH}$ coincided with $\mathrm{Ca}^{2+}$ entry. The scale of the acidification events was about $0.1 \mathrm{pH}$ unit, which is much smaller compared to acidification, caused by evoked depolarization (see Supplementary data) and corresponds well to previous observations [22]. Interestingly, even though addition of $\mathrm{KCl}$ causes continuous depolarization of the neuronal membrane, both calcium influx and acidification were transient, 
which proves that membrane depolarization is not enough to cause acidification, but rather neuronal activity is required. Although calcium entry seems to precede $\mathrm{pH}$ shifts, it is not clear whether calcium entry is a reason for cytoplasm acidification. A potential explanation that this acidification is caused by $\mathrm{Ca}^{2+} / \mathrm{H}^{+}$exchange involving $\mathrm{Ca}^{2+}$-ATPases is not supported as we did not observe an inhibitory effect of either $500 \mu \mathrm{M} \mathrm{La}^{3+}$, the inhibitor of L-type $\mathrm{Ca}^{2+}$-ATPases, or $10 \mu \mathrm{M}$ SERCA inhibitor thapsigargin (See supplementary data). Since during simultaneous recording of intracellular calcium level and $\mathrm{pH}$ the time lapse images were taken every 3 seconds, which was comparable to the duration of calcium entry (usually 3-5 seconds), we could not precisely resolve intracellular calcium elevations, therefore we could not evaluate whether the amplitude of calcium influx correlated with the amplitude of the following $\mathrm{pH}$-shift. We hypothesize that the oscillations might have metabolic nature, but this suggestion awaits to be proven experimentally.

In our experiments, basal $\mathrm{pH}_{\mathrm{i}}$ was found to be $\sim 7.4$. This result corresponds well to the mean values observed previously in rat hippocampal slices [22], although it is higher than the 6.8-7.2 values reported for other experimental systems, e.g., rat hippocampal neurons using pHluorin [40] and isolated snail neurons using a synthetic indicator [37]. This difference may stem from many factors such as difference in age and species of the donor animals (mouse E17 in our experiments and rat P1-2 in ref. [40]), different metabolic load, etc. However, it is clear that ratiometric measurements with a genetically encoded sensor followed by direct calibration are preferential compared to single-wavelength recordings of intensiometric probes where indirect estimations are necessary to calculate the $\mathrm{pH}$ values.

Neuronal cells are extremely polarized, with presynaptic and postsynaptic termini serving as the opposite "poles" of neurons. Being very small in volume, around $1 \mathrm{aL}[\underline{50}]$, these cytoplasmic subcompartments are the essence of signal transmission within the neuronal network. It must be noted that $\mathrm{pH}$ of 7.4 corresponds to $\sim 40 \mathrm{nM} \mathrm{H}^{+}$ions, which corresponds to less than a single free proton on average per $1 \mathrm{aL}$ terminal volume. Such values are extremely hard to measure using the sensor which shuffles freely within the cytoplasmic and synaptic compartments. But as these two types of synaptic termini are so different in function (release of synaptic vesicles content from presynaptic termini and reception, coupled with ion channels opening, in postsynaptic termini) one would expect that the dynamics and amplitude of $\mathrm{pH}$ changes may differ between the two compartments. The possibility of $\mathrm{pH}$-gradients in neurons was demonstrated previously [37], although no quantitative data on both pre- and postsynaptic $\mathrm{pH}$ changes in neuronal culture during spontaneous activity has been obtained so far.

The protein nature of SypHer-2 allowed us to improve the resolution of neuronal $\mathrm{pH}$ monitoring to the level of single pre- and postsynaptic termini. We were able to monitor $\mathrm{pH}$ changes 
in these two structures and revealed a two-fold difference in the amplitude of $\mathrm{pH}$ change: whereas presynaptic $\mathrm{pH}$ changes resembled those in the cytoplasm, with an amplitude of $\sim 0.1 \mathrm{pH}$ unit, postsynaptic $\mathrm{pH}$ changed almost 0.2 units on average during each spike. Simultaneous registration of intracellular $\mathrm{pH}$ and calcium level dynamics in postsynaptic compartments shows that higher amplitude of $\mathrm{pH}$ shift might be associated with prolonged calcium entry into spines (Supplementary figure 3), that likely causes higher metabolic load of the postsynapses. Previous analysis has shown that more than half of energy consumption in neurons is related to ion currents via NMDA receptors and postsynaptic potential modulation [18]. Another reason of the acidification dynamics difference might be that either the $\mathrm{pH}$-regulation system or $\mathrm{pH}$-buffering capacity is different in the cell body and presynaptic compartments versus postsynaptic compartments. As mentioned above, the dendritic spines are very small in volume and have a specific protein environment. Both hypotheses need further tests.

In summary, SypHer-2 allowed us to perform the first comparative quantitative measurements of $\mathrm{pH}$ in neuronal termini. Clearly, targeting the probe to various subcompartments by fusing it to particular proteins could vastly improve the capacity and the resolution of monitoring cellular activities.

Ratiometric readout of SypHer-2 is beneficial not only in imaging at the scale of subcellular compartments, but also at the tissue scale. To the best of our knowledge, $\mathrm{pH}$ dynamics during tail regeneration have previously been studied only by indirect approaches based on using membranevoltage-reporter dyes, under conditions of targeted modulation of V-ATPase activity [29-31] or using synthetic pH indicators BCECF and SNARF-5F [51]. By means of this approach, the authors obtained evidence of alkalinization of the cytoplasm of cells located next to the amputation site in the damaged tissue. However, the first time point of these experiments was 6 hours after tail amputation. In present work, by using SypHer-2 sensor, we have performed for the first time a direct measurement of $\mathrm{pH}$ in the tail cells within one hour after amputation. As a result, we were able to reveal a previously unknown reversible drop of $\mathrm{pH}$, which starts immediately following the amputation event and reaches minimal $\mathrm{pH}$ value as soon as 3-4 min post-amputation. Thus, this effect appears to be the earliest of all studied events that precede Xenopus tail regeneration [31].

The magnitude of this $\mathrm{pH}$ drop was the greatest in cells adjacent to the wound and gradually decreased as distance from the wound was increased. This suggests that the observed effect may be caused by some signal emitted by cells at the edge of amputation which weakens with distance from the edge. Recent studies show that $\mathrm{H}_{2} \mathrm{O}_{2}$ might be such a signal $[\underline{52}, \underline{53}]$. Meanwhile, at least under conditions of the $30 \mathrm{sec}$ gap between neighboring frames used in our time-lapse series, we 
were unable to reveal any time delay of the $\mathrm{pH}$ drop between cells at the amputation edge and those located far from this place. Given that the effect of $\mathrm{pH}$ drop was still detectable up to 1000 microns from the edge, one may estimate that such a signal, be it $\mathrm{H}_{2} \mathrm{O}_{2}$ or something else, should spread with velocity not less than 30 microns/sec. Further experiments are necessary to test this prediction.

It is known that in the intact tail cells, ATP-dependent $\mathrm{H}^{+}$pumps are responsible for the removal of $\mathrm{H}^{+}$ions from the cytoplasm. As a result, a $\mathrm{pH}$ gradient between the cytoplasm and intercellular spaces is established. One may speculate that the signal induced by amputation leads to a blockade of these $\mathrm{H}^{+}$pumps, which in turn elicits acidification of the cytoplasm because of $\mathrm{H}^{+}$ influx along the gradient. Indeed, we have observed reduction of acidification amplitude by replacing the external medium to slightly more alkaline one, reducing the $\mathrm{H}^{+}$gradient across the plasma membrane of the cells. In turn, our observation that the return of $\mathrm{pH}$ to its initial values depends on the activity of ATP-dependent $\mathrm{H}^{+}$pumps, may indicate that such a signal, which blocks $\mathrm{H}^{+}$pumps' activity, might have a temporary character and vanish soon after amputation. Alternatively, this signal could elicit temporary opening of additional $\mathrm{H}^{+}$gates in cell membranes, leading to an increase of intracellular $\mathrm{pH}$. Obviously, additional experiments are necessary to sort between these and other possible mechanisms.

It has been shown recently that activation of V-ATPase-based $\mathrm{H}^{+}$efflux from the regeneration bud cells, which is registered $6 \mathrm{~h}$ after tail amputation, is critical for its regeneration [29, 30]. We have now found that, conversely, decreasing the magnitude of the intracellular $\mathrm{pH}$ drop by applying alkaline solution immediately after the tail amputation leads to a reduction in the regeneration capability. Besides, we have observed similar diminishing of the $\mathrm{pH}$ drop depth in tails amputated during the refractory period, the stage of development when the regeneration ability is lost. Though these particular experiments have demonstrated no direct causal relationship between this $\mathrm{pH}$ drop and subsequent regeneration, their results hint at a possibility of such a relationship, which could be tested in further experiments.

Speculating about mechanisms by which the $\mathrm{pH}$ drop could influence regeneration, one may suppose that acidification can trigger some intracellular pathways. There are reports, for example, indicating that a decrease of the intracellular $\mathrm{pH}$ may cause stimulating effects on BMP and Wnt signaling, which in turn are known to regulate the expression of many target genes involved in regeneration (see, for example, [노, $\underline{55}]$ ).

Taken together, our results show that SypHer-2 has a superior performance in the biological imaging models of various scales. Its ratiometric response and improved brightness provide quantitative information on $\mathrm{pH}$ changes in the living cells, subcellular compartments, and tissues. 


\section{Acknowledgements.}

This work was supported by the Russian foundation for basic research (13-04-40333- $\mathrm{H}$ to V.V.B, 13-04-40332-H to P.M.B, 13-04-01516-A and 13-04-40194-H to A.G.Z., 13-04-00352 to E.S.N); Molecular and Cell Biology program of Russian Academy of Sciences (V.V.B \& E.S.N); CREST, JST and Grants-in-Aid for Scientific Research (26250014), JAPAN to S.O.; National Institute of Aging (R01AG040209 to G.E.); NYSTEM (to G.E); Russian science foundation (14-1400557 to A.G.Z, experiments with time-lapse SypHer2 ratio measurements using M205 stereomicroscope), and the Russian Ministry of Education andScience (11.G34.31.0071) to G.E.

\section{FIGURE LEGENDS}

Fig. 1. SypHer-2 vs. SypHer pH sensitivity and brightness in cells. A) Calibration of SypHer-2 in HeLa-Kyoto cells with ionophore-containing buffers. The signal was measured as a ratio between intensities of fluorescence excited by $504 \mathrm{~nm}$ (middle row) and $427 \mathrm{~nm}$ (upper row) light. Numbers represent calibration buffer $\mathrm{pH}$, scale bar 25 is $\mu \mathrm{m}$. Lookup table reflects SypHer-2 ratio. B) Calibration curves for SypHer (black) and SypHer-2 (red). At each pH point mean fluorescence excitation ratio was measured for at least 20 HeLa cells. C), D) Brightness of SypHer-2 vs. SypHer fluorescence excited by $427 \mathrm{~nm}$ (light grey) and $504 \mathrm{~nm}$ (dark grey) light in NIH/3T3 (C) and HeLaKyoto (D) cells. Fluorescence intensities were collected for at least 15 cells for each line and normalized using mean SypHer fluorescence at $504 \mathrm{~nm}$ excitation. Error bars indicate 2xSEM. $18 \mathrm{~h}$ after transfection with SypHer or SypHer-2 plasmids, cells were incubated with $60 \mu \mathrm{M}$ protein synthesis inhibitor cycloheximide ( $\mathrm{CHX}$ ) for $1 \mathrm{~h}$ and mean fluorescence from 20-30 cells, excited at $504 \mathrm{~nm}$, was collected every hour. Error bars indicate 2xSEM.

Fig. 2. SypHer-2 detects activity-associated pH oscillations in a dissociated primary neuronal culture. A) Time lapse imaging of a cultured mouse hippocampal neuron using SypHer-2 (upper row) for $\mathrm{pH}$ and R-GECO (lower row) for calcium measurements. Numbers indicate time in seconds, lookup table reflects SypHer-2 ratio. Scale bar is $40 \mu \mathrm{m}$; B), C) Intracellular $\mathrm{pH}$ and calcium levels were registered in neuronal cell bodies using SypHer-2 (black) and R-GECO (red) respectively. $\mathrm{pH}$ oscillations were associated with calcium entry into cells, and both were inhibited in the presence of $1 \mathrm{mM}$ BAPTA-AM (B) or $100 \mathrm{nM}$ TTX (C); D) After each experiment SypHer-2 ratiometric signal was calibrated using ionophore containing buffers. Numbers indicate $\mathrm{pH}$ values; 
E) Dynamics of intracellular synthetic indicator BCECF-AM fluorescence, excited by $504 \mathrm{~nm}$ light, confirms existence of $\mathrm{pH}$ oscillations. All experiments were replicated with 3 to 5 neuronal cells from two different preparations.

Fig. 3. SypHer-2 fused with Homer and Synaptophysin allows pH detection in synaptic structures. A) Expression of SypHer-2-Homer and Synaptophysin-SypHer-2 in cultured mouse hippocampal neurons; $\mathrm{B}) \mathrm{pH}$ oscillations registered in a single postsynaptic terminus using the SypHer-2-Homer; C) pH oscillations registered in a single presynaptic bouton using SynaptophysinSypHer-2; D) amplitude of $\mathrm{pH}$ oscillations in presynaptic vs. postsynaptic termini. All experiments were replicated with 3 to 5 neuronal cells from two different preparations. Scale bars are $40 \mu \mathrm{m}$.

Fig. 4. Intracellular pH drop in the tail of Xenopus laevis tadpole after amputation.

A) Typical dynamics of mean $\mathrm{pH}$ value changes as measured in ten transverse rectangular frames before amputation and during one hour after it (see Materials and Methods for details). Each of dynamics curve is marked by a specific color, beginning from the black color, which is assigned to the frame nearest to the amputation edge. The position of each frame from the amputation edge to the median of the frame is indicated in microns. Inset image shows the tip of the same regenerating tail after 3 days of amputation. An example of the amputated tail in pseudocolors corresponding to SypHer-2 ratio, with one measuring frame located at position of $75 \mu \mathrm{m}$ from the amputation edge is shown on the right. Note that the blue color on the sides of the tail is a specific characteristic of this particular tail in which the injected SypHer-2 mRNA is absent in cells of the lateral sides of the tail. Due to a low auto-fluorescence of the tail tissues the ratio of fluorescence intensities in the lateral regions appears to be different from zero and appears as a constant blue pseudocolor. Of note, the auto-fluorescence intensity is more than 10-fold lower than the intensity of signal generated even by low amount of SypHer-2; B) Mean value of $\mathrm{pH}$ near the amputation edge (measuring frame with the median at $15 \mu \mathrm{m}$ from the edge) before amputation and in the minimum of $\mathrm{pH}$ dynamics curve of stage 42 tails (left pair of bars), stage 42 tails of tadpoles treated by pH 8.2 solution for $2 \mathrm{~h}$ before amputation (middle pair of bars) and stage 46 tails (right pair of bars). Standard deviation is indicated for each bar. The numbers show total number of tails in which $\mathrm{pH}$ was measured at the level of 15 microns from the amputation edge before and after amputation.; C) Representative dynamics of mean $\mathrm{pH}$ value measured as described in $\mathrm{A}$, but in tails of stage 42 tadpoles treated with a V-ATPase inhibitor bafilomycin (200 $\mathrm{nM}$ for $1 \mathrm{~h}$ before amputation); D) Representative dynamics of mean $\mathrm{pH}$ value measured as described in $\mathrm{A}$, but in tails of stage 42 tadpoles treated by $\mathrm{pH} 8.2$ solution for $2 \mathrm{~h}$ before amputation. Inset image shows tip of the same tail after 3 days of 
amputation; E) Representative dynamics of mean $\mathrm{pH}$ value measured as described in $\mathrm{A}$, but in tails of stage 46 tadpoles. Inset image shows tip of the same tail after 3 days of amputation.

\section{References}

[1] R. Bizzarri, M. Serresi, S. Luin, F. Beltram, Green fluorescent protein based pH indicators for in vivo use: a review, Analytical and bioanalytical chemistry, 393 (2009) 1107-1122.

[2] J. Han, K. Burgess, Fluorescent indicators for intracellular pH, Chemical reviews, 110 (2010) 2709-2728.

[3] M. Bencina, Illumination of the spatial order of intracellular $\mathrm{pH}$ by genetically encoded $\mathrm{pH}$ sensitive sensors, Sensors (Basel), 13 (2013) 16736-16758.

[4] R.Y. Tsien, T. Pozzan, T.J. Rink, T-cell mitogens cause early changes in cytoplasmic free Ca2+ and membrane potential in lymphocytes, Nature, 295 (1982) 68-71.

[5] K.J. Buckler, R.D. Vaughan-Jones, Application of a new pH-sensitive fluoroprobe (carboxySNARF-1) for intracellular $\mathrm{pH}$ measurement in small, isolated cells, Pflugers Archiv : European journal of physiology, 417 (1990) 234-239.

[6] S. Sankaranarayanan, D. De Angelis, J.E. Rothman, T.A. Ryan, The use of pHluorins for optical measurements of presynaptic activity, Biophysical journal, 79 (2000) 2199-2208.

[7] G.T. Hanson, T.B. McAnaney, E.S. Park, M.E. Rendell, D.K. Yarbrough, S. Chu, L. Xi, S.G. Boxer, M.H. Montrose, S.J. Remington, Green fluorescent protein variants as ratiometric dual emission $\mathrm{pH}$ sensors. 1. Structural characterization and preliminary application, Biochemistry, 41 (2002) 15477-15488.

[8] R. Bizzarri, C. Arcangeli, D. Arosio, F. Ricci, P. Faraci, F. Cardarelli, F. Beltram, Development of a novel GFP-based ratiometric excitation and emission $\mathrm{pH}$ indicator for intracellular studies, Biophysical journal, 90 (2006) 3300-3314. 
[9] M. Tantama, Y.P. Hung, G. Yellen, Imaging intracellular $\mathrm{pH}$ in live cells with a genetically encoded red fluorescent protein sensor, Journal of the American Chemical Society, 133 (2011) 10034-10037.

[10] Y. Li, R.W. Tsien, pHTomato, a red, genetically encoded indicator that enables multiplex interrogation of synaptic activity, Nature neuroscience, 15 (2012) 1047-1053.

[11] K. Nehrke, Intracellular $\mathrm{pH}$ measurements in vivo using green fluorescent protein variants, Methods Mol Biol, 351 (2006) 223-239.

[12] D. Poburko, J. Santo-Domingo, N. Demaurex, Dynamic regulation of the mitochondrial proton gradient during cytosolic calcium elevations, The Journal of biological chemistry, 286 (2011) $11672-11684$.

[13] V.V. Belousov, A.F. Fradkov, K.A. Lukyanov, D.B. Staroverov, K.S. Shakhbazov, A.V. Terskikh, S. Lukyanov, Genetically encoded fluorescent indicator for intracellular hydrogen peroxide, Nat Methods, 3 (2006) 281-286.

[14] K.N. Markvicheva, D.S. Bilan, N.M. Mishina, A.Y. Gorokhovatsky, L.M. Vinokurov, S. Lukyanov, V.V. Belousov, A genetically encoded sensor for $\mathrm{H} 2 \mathrm{O} 2$ with expanded dynamic range, Bioorganic \& medicinal chemistry, 19 (2011) 1079-1084.

[15] K.R. Hoyt, S.R. Arden, E. Aizenman, I.J. Reynolds, Reverse Na+/Ca2+ exchange contributes to glutamate-induced intracellular $\mathrm{Ca2}+$ concentration increases in cultured rat forebrain neurons, Molecular pharmacology, 53 (1998) 742-749.

[16] C.R. Rose, B.R. Ransom, Regulation of intracellular sodium in cultured rat hippocampal neurones, The Journal of physiology, 499 ( Pt 3) (1997) 573-587.

[17] M. Mukhtarov, L. Liguori, T. Waseem, F. Rocca, S. Buldakova, D. Arosio, P. Bregestovski, Calibration and functional analysis of three genetically encoded $\mathrm{Cl}(-) / \mathrm{pH}$ sensors, Frontiers in molecular neuroscience, 6 (2013) 9.

[18] J.J. Harris, R. Jolivet, D. Attwell, Synaptic energy use and supply, Neuron, 75 (2012) $762-$ 777.

[19] S. Trapp, M. Luckermann, P.A. Brooks, K. Ballanyi, Acidosis of rat dorsal vagal neurons in situ during spontaneous and evoked activity, The Journal of physiology, 496 ( Pt 3) (1996) 695-710. 
[20] R.Z. Zhan, N. Fujiwara, E. Tanaka, K. Shimoji, Intracellular acidification induced by membrane depolarization in rat hippocampal slices: roles of intracellular $\mathrm{Ca} 2+$ and glycolysis, Brain research, 780 (1998) 86-94.

[21] M.L. Wu, J.H. Chen, W.H. Chen, Y.J. Chen, K.C. Chu, Novel role of the Ca(2+)-ATPase in NMDA-induced intracellular acidification, The American journal of physiology, 277 (1999) C717727.

[22] J.V. Raimondo, A. Irkle, W. Wefelmeyer, S.E. Newey, C.J. Akerman, Genetically encoded proton sensors reveal activity-dependent $\mathrm{pH}$ changes in neurons, Frontiers in molecular neuroscience, 5 (2012) 68.

[23] M. Pasternack, C. Bountra, J. Voipio, K. Kaila, Influence of extracellular and intracellular pH on GABA-gated chloride conductance in crayfish muscle fibres, Neuroscience, 47 (1992) 921 929.

[24] G.C. Tombaugh, G.G. Somjen, Differential sensitivity to intracellular pH among high- and low-threshold Ca2+ currents in isolated rat CA1 neurons, Journal of neurophysiology, 77 (1997) 639-653.

[25] Y.H. Chen, M.L. Wu, W.M. Fu, Regulation of presynaptic NMDA responses by external and intracellular $\mathrm{pH}$ changes at developing neuromuscular synapses, The Journal of neuroscience : the official journal of the Society for Neuroscience, 18 (1998) 2982-2990.

[26] R.D. Blakely, R.H. Edwards, Vesicular and plasma membrane transporters for neurotransmitters, Cold Spring Harbor perspectives in biology, 4 (2012).

[27] Z.Q. Xiong, P. Saggau, J.L. Stringer, Activity-dependent intracellular acidification correlates with the duration of seizure activity, The Journal of neuroscience : the official journal of the Society for Neuroscience, 20 (2000) 1290-1296.

[28] K.F. Lee, C. Soares, J.C. Beique, Examining form and function of dendritic spines, Neural plasticity, 2012 (2012) 704103.

[29] D.S. Adams, A. Masi, M. Levin, H+ pump-dependent changes in membrane voltage are an early mechanism necessary and sufficient to induce Xenopus tail regeneration, Development, 134 (2007) 1323-1335. 
[30] A.S. Tseng, M. Levin, Transducing bioelectric signals into epigenetic pathways during tadpole tail regeneration, Anatomical record, 295 (2012) 1541-1551.

[31] A.S. Tseng, M. Levin, Tail regeneration in Xenopus laevis as a model for understanding tissue repair, Journal of dental research, 87 (2008) 806-816.

[32] S. Okabe, T. Urushido, D. Konno, H. Okado, K. Sobue, Rapid redistribution of the postsynaptic density protein PSD-Zip45 (Homer 1c) and its differential regulation by NMDA receptors and calcium channels, The Journal of neuroscience : the official journal of the Society for Neuroscience, 21 (2001) 9561-9571.

[33] S. Okabe, A. Miwa, H. Okado, Spine formation and correlated assembly of presynaptic and postsynaptic molecules, The Journal of neuroscience : the official journal of the Society for Neuroscience, 21 (2001) 6105-6114.

[34] M.L. Seibenhener, M.W. Wooten, Isolation and culture of hippocampal neurons from prenatal mice, Journal of visualized experiments : JoVE, (2012).

[35] G.V. Ermakova, E.A. Solovieva, N.Y. Martynova, A.G. Zaraisky, The homeodomain factor Xanf represses expression of genes in the presumptive rostral forebrain that specify more caudal brain regions, Developmental biology, 307 (2007) 483-497.

[36] D.S. Bilan, L. Pase, L. Joosen, A.Y. Gorokhovatsky, Y.G. Ermakova, T.W. Gadella, C. Grabher, C. Schultz, S. Lukyanov, V.V. Belousov, HyPer-3: a genetically encoded $\mathrm{H}(2) \mathrm{O}(2)$ probe with improved performance for ratiometric and fluorescence lifetime imaging, ACS chemical biology, 8 (2013) 535-542.

[37] C.J. Schwiening, D. Willoughby, Depolarization-induced pH microdomains and their relationship to calcium transients in isolated snail neurones, The Journal of physiology, 538 (2002) 371-382.

[38] N. Svichar, S. Esquenazi, H.Y. Chen, M. Chesler, Preemptive regulation of intracellular $\mathrm{pH}$ in hippocampal neurons by a dual mechanism of depolarization-induced alkalinization, The Journal of neuroscience : the official journal of the Society for Neuroscience, 31 (2011) 6997-7004.

[39] D. Willoughby, C.J. Schwiening, Electrically evoked dendritic $\mathrm{pH}$ transients in rat cerebellar Purkinje cells, The Journal of physiology, 544 (2002) 487-499. 
[40] V. Rangaraju, N. Calloway, T.A. Ryan, Activity-driven local ATP synthesis is required for synaptic function, Cell, 156 (2014) 825-835.

[41] M. Chiappalone, M. Bove, A. Vato, M. Tedesco, S. Martinoia, Dissociated cortical networks show spontaneously correlated activity patterns during in vitro development, Brain research, 1093 (2006) 41-53.

[42] Y. Zhao, S. Araki, J. Wu, T. Teramoto, Y.F. Chang, M. Nakano, A.S. Abdelfattah, M. Fujiwara, T. Ishihara, T. Nagai, R.E. Campbell, An expanded palette of genetically encoded $\mathrm{Ca}(2)(+)$ indicators, Science, 333 (2011) 1888-1891.

[43] G.J. Augustine, F. Santamaria, K. Tanaka, Local calcium signaling in neurons, Neuron, 40 (2003) 331-346.

[44] T. Ebihara, I. Kawabata, S. Usui, K. Sobue, S. Okabe, Synchronized formation and remodeling of postsynaptic densities: long-term visualization of hippocampal neurons expressing postsynaptic density proteins tagged with green fluorescent protein, The Journal of neuroscience : the official journal of the Society for Neuroscience, 23 (2003) 2170-2181.

[45] C.W. Beck, B. Christen, J.M. Slack, Molecular pathways needed for regeneration of spinal cord and muscle in a vertebrate, Developmental cell, 5 (2003) 429-439.

[46] M.J. Mahon, pHluorin2: an enhanced, ratiometric, $\mathrm{pH}$-sensitive green florescent protein, Advances in bioscience and biotechnology, 2 (2011) 132-137.

[47] G. Miesenbock, D.A. De Angelis, J.E. Rothman, Visualizing secretion and synaptic transmission with pH-sensitive green fluorescent proteins, Nature, 394 (1998) 192-195.

[48] M.D. Corporation, The Axon CNS Guide to Electrophysiology \& Biophysics Laboratory Techniques, Molecular Devices, 2006.

[49] G.E. Hardingham, Y. Fukunaga, H. Bading, Extrasynaptic NMDARs oppose synaptic NMDARs by triggering CREB shut-off and cell death pathways, Nature neuroscience, 5 (2002) 405414.

[50] K.M. Harris, J.K. Stevens, Dendritic spines of CA 1 pyramidal cells in the rat hippocampus: serial electron microscopy with reference to their biophysical characteristics, The Journal of neuroscience : the official journal of the Society for Neuroscience, 9 (1989) 2982-2997. 
[51] D.S. Adams, A.S. Tseng, M. Levin, Light-activation of the Archaerhodopsin $\mathrm{H}(+)$-pump reverses age-dependent loss of vertebrate regeneration: sparking system-level controls in vivo, Biology open, 2 (2013) 306-313.

[52] P. Niethammer, C. Grabher, A.T. Look, T.J. Mitchison, A tissue-scale gradient of hydrogen peroxide mediates rapid wound detection in zebrafish, Nature, 459 (2009) 996-999.

[53] N.R. Love, Y. Chen, S. Ishibashi, P. Kritsiligkou, R. Lea, Y. Koh, J.L. Gallop, K. Dorey, E. Amaya, Amputation-induced reactive oxygen species are required for successful Xenopus tadpole tail regeneration, Nature cell biology, 15 (2013) 222-228.

[54] J.A. Uzman, S. Patil, A.R. Uzgare, A.K. Sater, The role of intracellular alkalinization in the establishment of anterior neural fate in Xenopus, Developmental biology, 193 (1998) 10-20.

[55] C.M. Cruciat, B. Ohkawara, S.P. Acebron, E. Karaulanov, C. Reinhard, D. Ingelfinger, M. Boutros, C. Niehrs, Requirement of prorenin receptor and vacuolar H+-ATPase-mediated acidification for Wnt signaling, Science, 327 (2010) 459-463. 
B

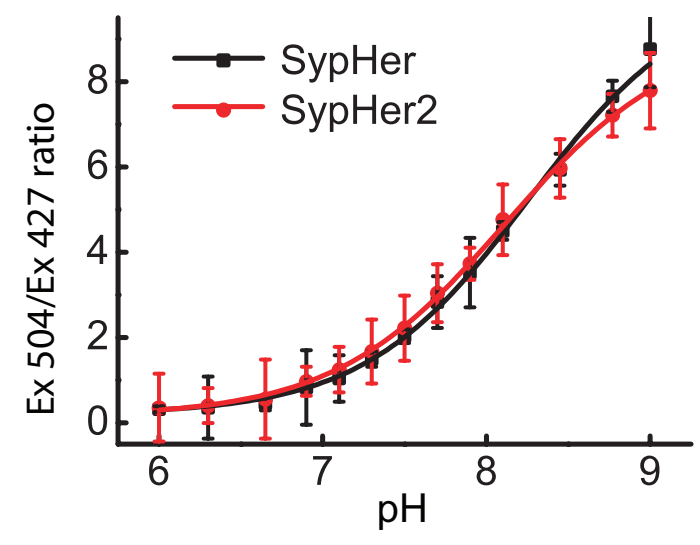

$\mathrm{D}$

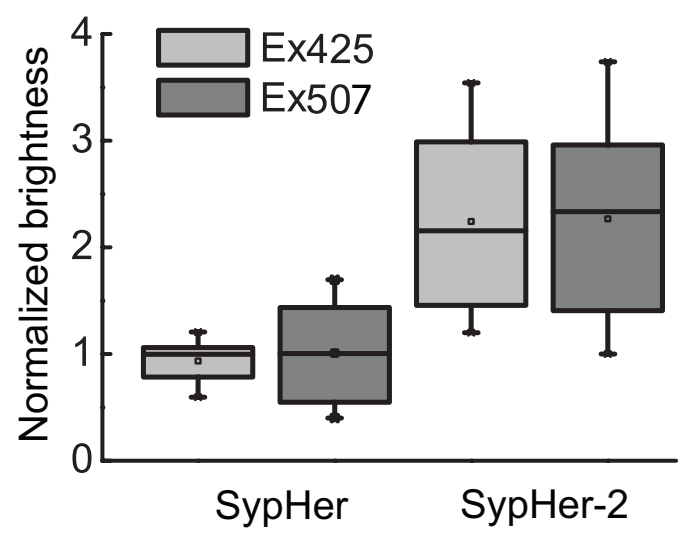

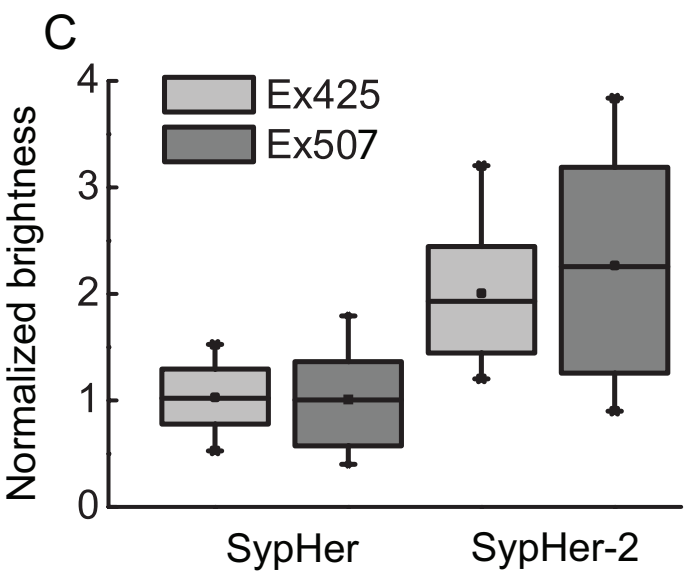

$\mathrm{E}$

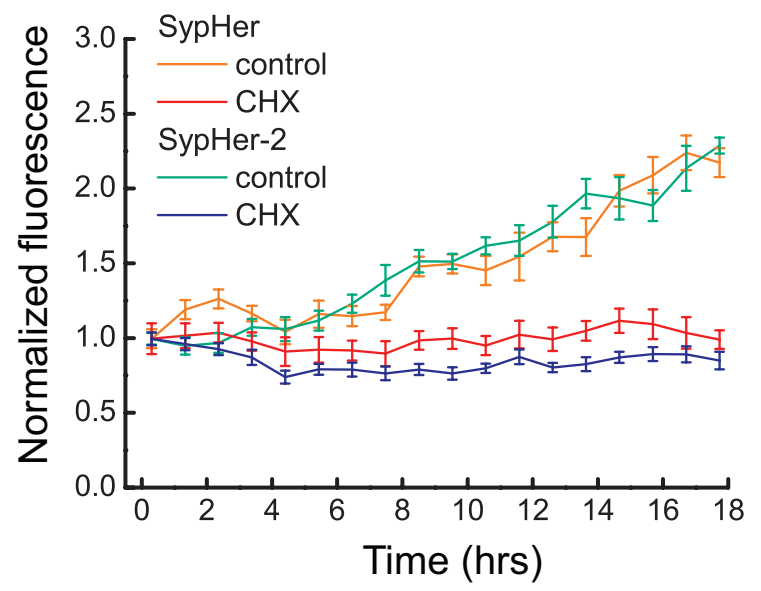

Fig. 1. SypHer-2 vs. SypHer pH sensitivity and brightness in cells. A) Calibration of SypHer2 in HeLa-Kyoto cells with ionophore-containing buffers. The signal was measured as a ratio between intensities of fluorescence excited by $504 \mathrm{~nm}$ (middle row) and $427 \mathrm{~nm}$ (upper row) light. Numbers represent calibration buffer $\mathrm{pH}$, scale bar 25 is $\mu \mathrm{m}$. Lookup table reflects SypHer-2 ratio. B) Calibration curves for SypHer (black) and SypHer-2 (red). At each pH point mean fluorescence excitation ratio was measured for at least $20 \mathrm{HeLa}$ cells. C), D) Brightness of SypHer-2 vs. SypHer fluorescence excited by $427 \mathrm{~nm}$ (light grey) and $504 \mathrm{~nm}$ (dark grey) light in NIH/3T3 (C) and HeLa-Kyoto (D) cells. Fluorescence intensities were collected for at least 15 cells for each line and normalized using mean SypHer fluorescence at $504 \mathrm{~nm}$ excitation. Error bars indicate 2xSEM. $18 \mathrm{~h}$ after transfection with SypHer or SypHer-2 plasmids, cells were incubated with $60 \mu \mathrm{M}$ protein synthesis inhibitor cycloheximide (CHX) for $1 \mathrm{~h}$ and mean fluorescence from 20-30 cells, excited at 504 nm, was collected every hour. Error bars indicate 2xSEM. 

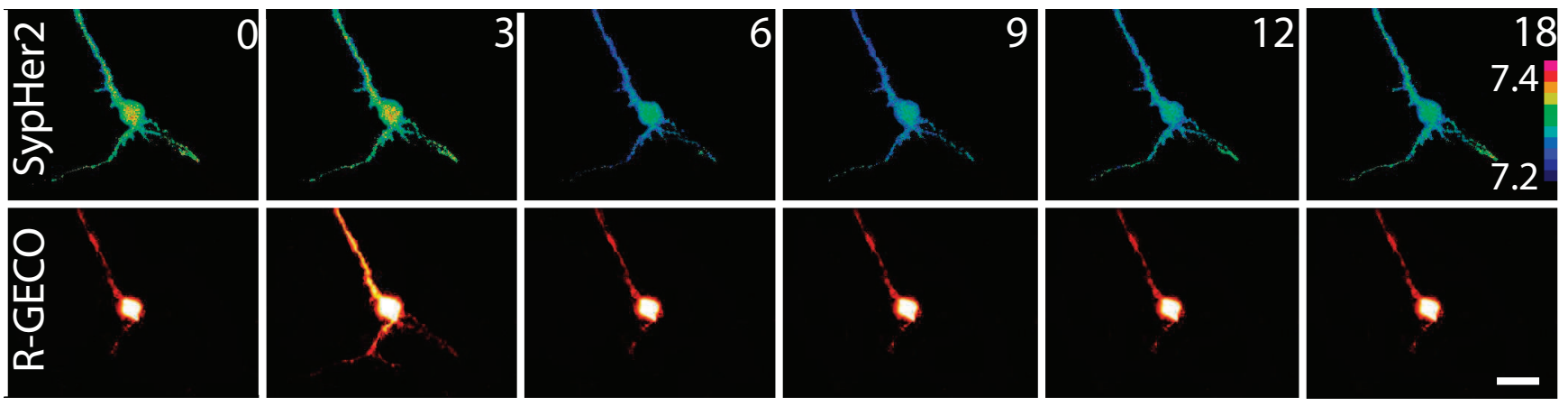

B

C
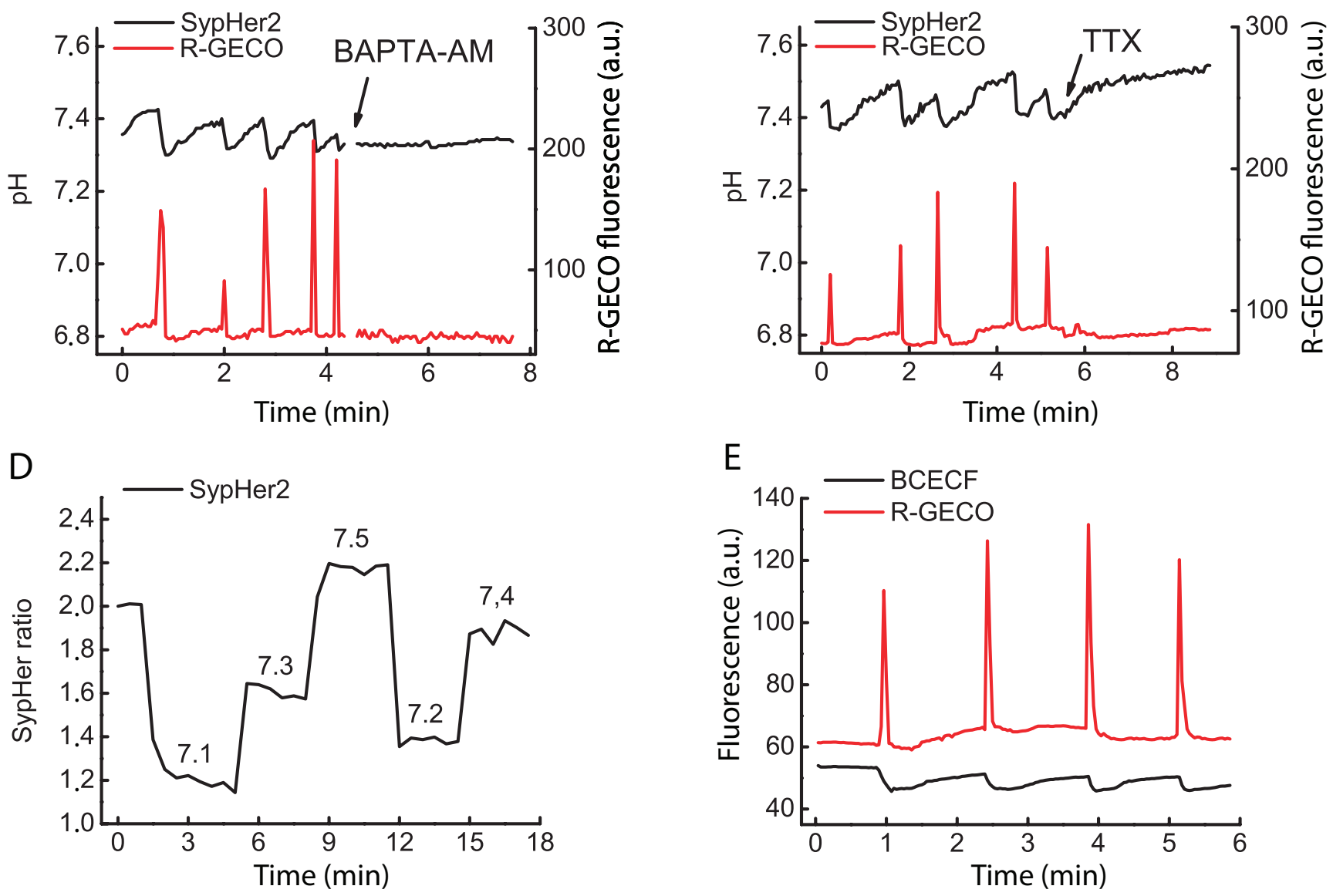

Fig. 2. SypHer-2 detects activity-associated pH oscillations in a dissociated primary

neuronal culture. A) Time lapse imaging of a cultured mouse hippocampal neuron using SypHer-2 (upper row) for $\mathrm{pH}$ and R-GECO (lower row) for calcium measurements. Numbers indicate time in seconds, lookup table reflects SypHer-2 ratio. Scale bar is $40 \mu \mathrm{m}$; B), C) Intracellular $\mathrm{pH}$ and calcium levels were registered in neuronal cell bodies using SypHer-2 (black) and R-GECO (red) respectively. $\mathrm{pH}$ oscillations were associated with calcium entry into cells, and both were inhibited in the presence of $1 \mathrm{mM}$ BAPTA-AM (B) or $100 \mathrm{nM}$ TTX (C); D) After each experiment SypHer-2 ratiometric signal was calibrated using ionophore containing buffers. Numbers indicate pH values; E) Dynamics of intracellular synthetic indicator BCECFAM fluorescence, excited by $504 \mathrm{~nm}$ light, confirms existence of $\mathrm{pH}$ oscillations. All experiments were replicated with 3 to 5 neuronal cells from two different preparations. 
A

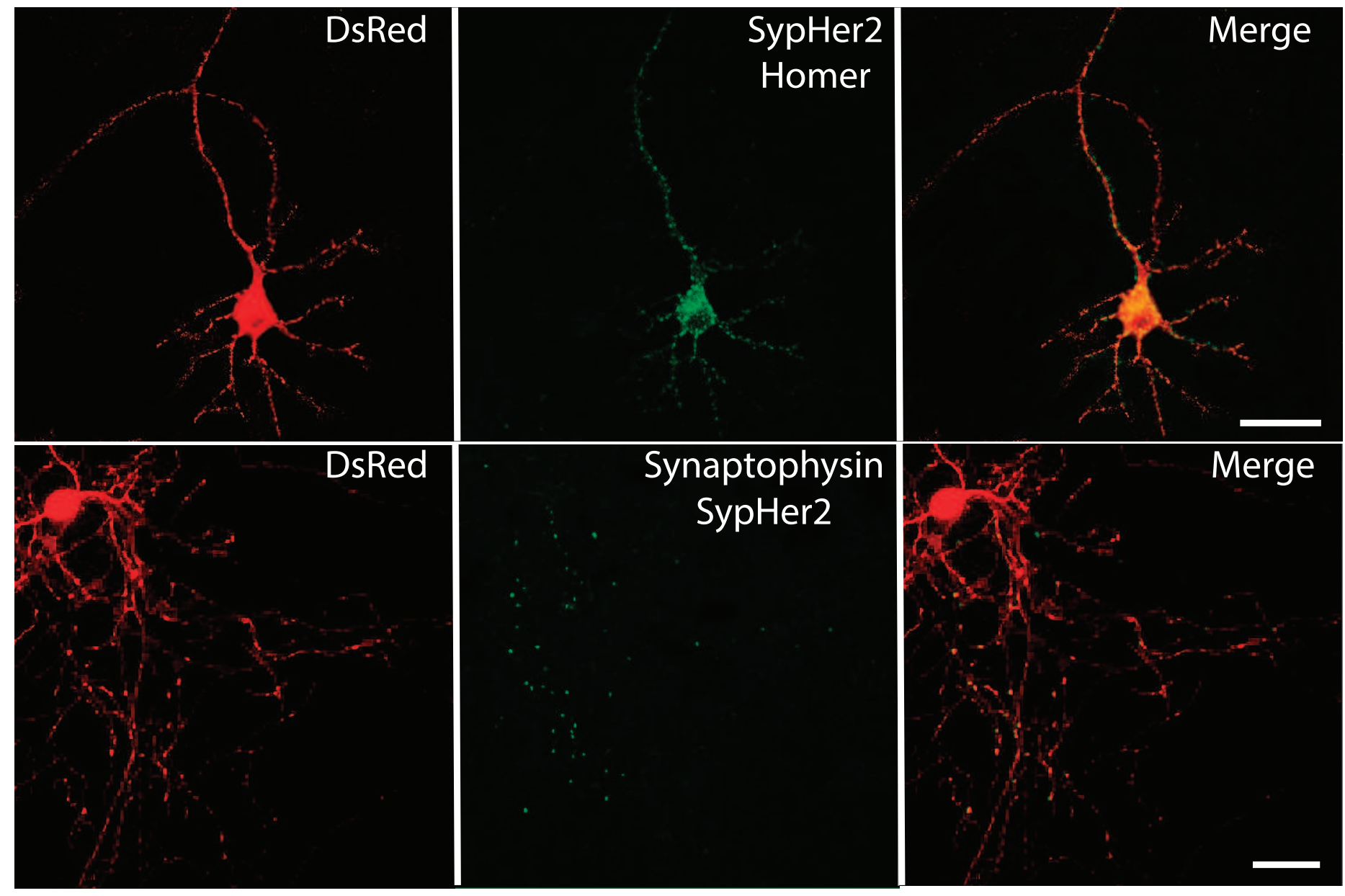

$\mathrm{B}$

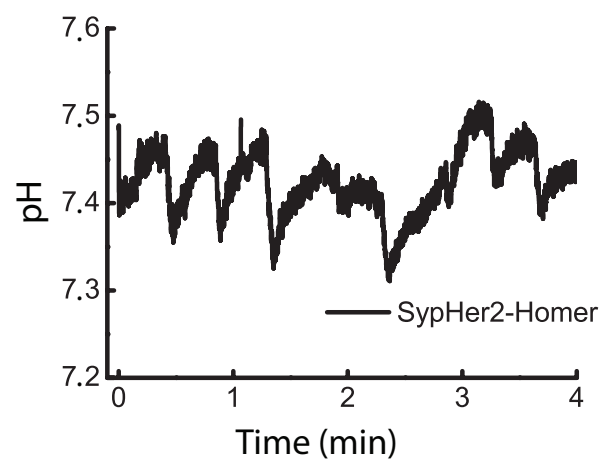

C

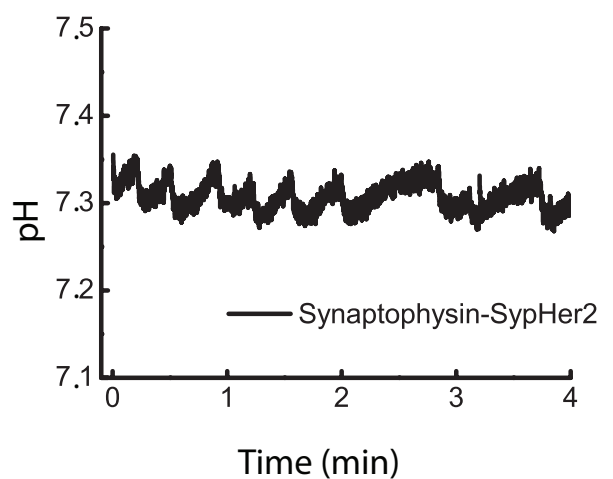

$\mathrm{D}$

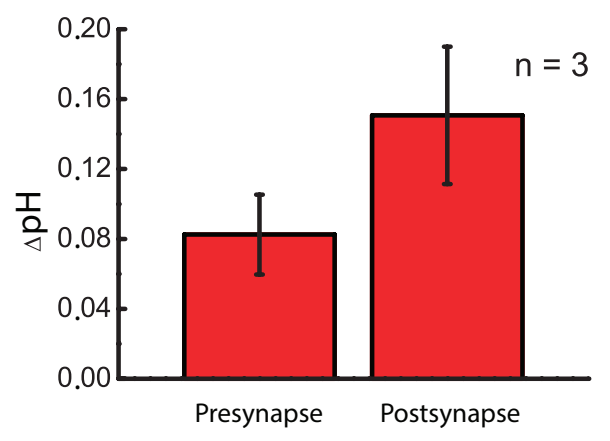

Fig. 3. SypHer-2 fused with Homer and Synaptophysin allows pH detection in synaptic structures. A) Expression of SypHer-2-Homer and Synaptophysin-SypHer-2 in cultured mouse hippocampal neurons; $\mathrm{B}) \mathrm{pH}$ oscillations registered in a single postsynaptic terminus using the SypHer-2-Homer; C) $\mathrm{pH}$ oscillations registered in a single presynaptic bouton using Synaptophysin-SypHer-2; D) amplitude of $\mathrm{pH}$ oscillations in presynaptic vs. postsynaptic termini. All experiments were replicated with 3 to 5 neuronal cells from two different preparations. Scale bars are $40 \mu \mathrm{m}$. 


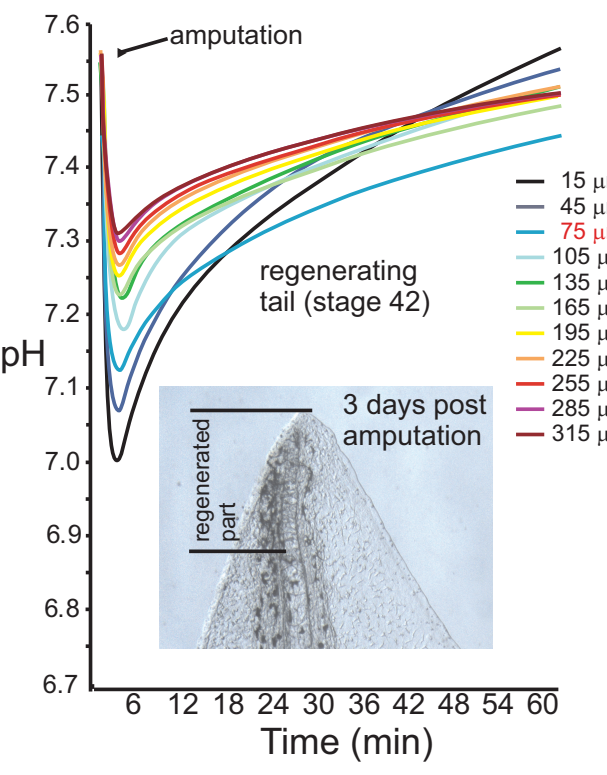

C

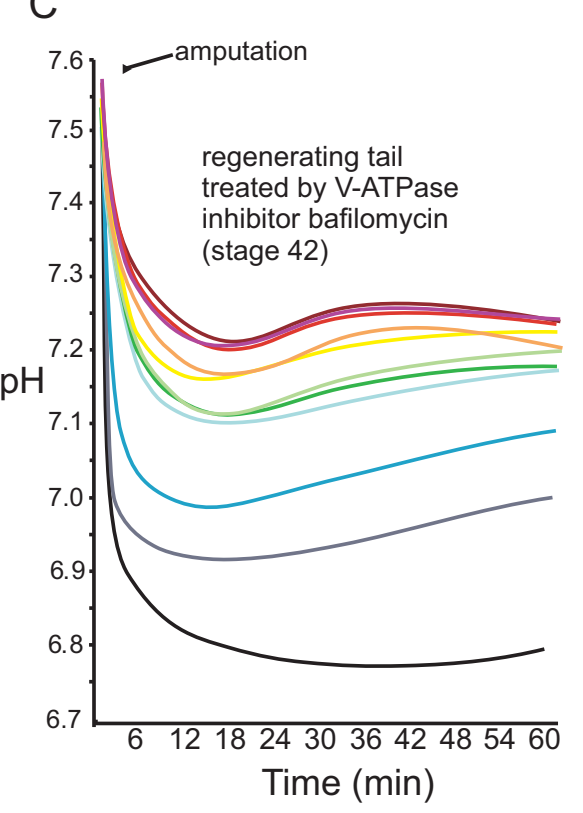

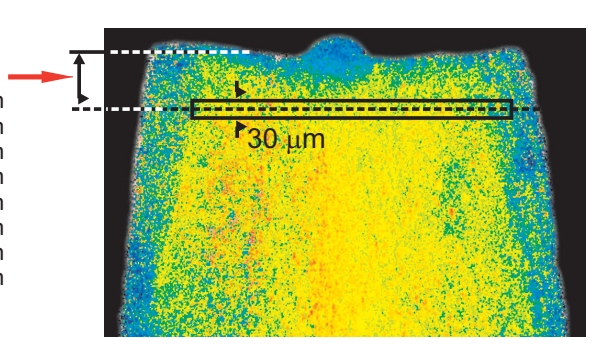
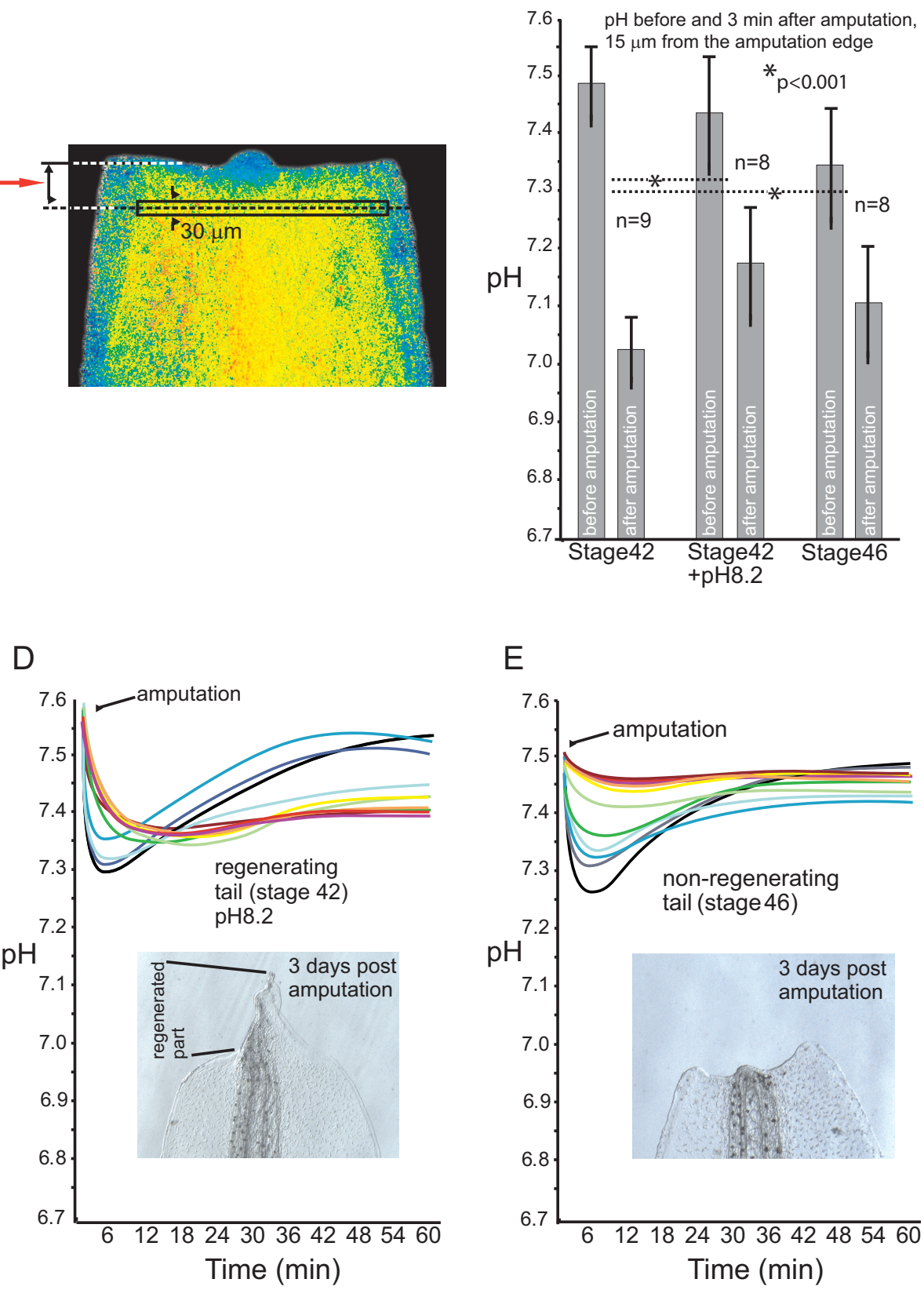

E

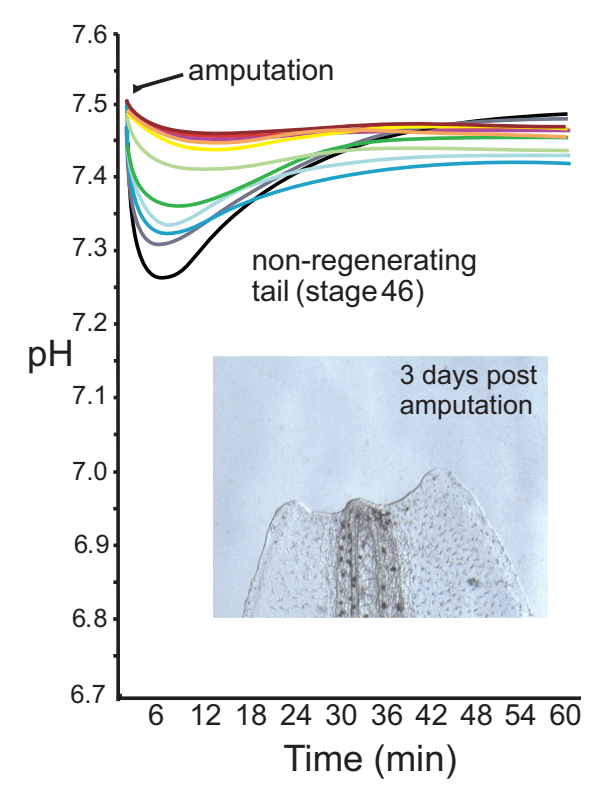

Fig. 4. Intracellular pH drop in the tail of Xenopus laevis tadpole after amputation.

A) Typical dynamics of mean $\mathrm{pH}$ value changes as measured in ten transverse rectangular frames before amputation and during one hour after it (see Materials and Methods for details). Each of dynamics curve is marked by a specific color, beginning from the black color, which is assigned to the frame nearest to the amputation edge. The position of each frame from the amputation edge to the median of the frame is indicated in microns. Inset image shows the tip of the same regenerating tail after 3 days of amputation. An example of the amputated tail in pseudocolors corresponding to SypHer-2 ratio, with one measuring frame located at position of $75 \mu \mathrm{m}$ from the amputation edge is shown on the right. Note that the blue color on the sides of the tail is a specific characteristic of this particular tail in which the injected SypHer-2 mRNA is absent in cells of the lateral sides of the tail. Due to a low autofluorescence of the tail tissues the ratio of fluorescence intensities in the lateral regions appears to be different from zero and appears as a constant blue pseudocolor. Of note, the auto-fluorescence intensity is more than 10-fold lower than the intensity of signal generated even by low amount of SypHer-2; B) Mean value of $\mathrm{pH}$ near the amputation edge (measuring frame with the median at $15 \mu \mathrm{m}$ from the edge) before amputation and in the minimum of $\mathrm{pH}$ dynamics curve of stage 42 tails (left pair of bars), stage 42 tails of tadpoles treated by $\mathrm{pH} 8.2$ solution for $2 \mathrm{~h}$ before amputation (middle pair of bars) and stage 46 tails (right pair of bars). Standard deviation is indicated for each bar. The numbers show total number of tails in which $\mathrm{pH}$ was measured at the level of 15 microns from the amputation edge before and after amputation.; C) Representative dynamics of mean $\mathrm{pH}$ value measured as described in $\mathrm{A}$, but in tails of stage 42 tadpoles treated with a V-ATPase inhibitor bafilomycin (200 $\mathrm{nM}$ for $1 \mathrm{~h}$ before amputation); D) Representative dynamics of mean $\mathrm{pH}$ value measured as described in A, but in tails of stage 42 tadpoles treated by $\mathrm{pH} 8.2$ solution for $2 \mathrm{~h}$ before amputation. Inset image shows tip of the same tail after 3 days of amputation; E) Representative dynamics of mean $\mathrm{pH}$ value measured as described in $\mathrm{A}$, but in tails of stage 46 tadpoles. Inset image shows tip of the same tail after 3 days of amputation. 